\title{
Identifikation der Einflussgrößen für die Auslegung eines Trennapparates zur selektiven Störstoffabscheidung bei der Co-Vergärung von organischen Reststoffen in Abwasserreinigungsanlagen
}

\author{
Thomas Senfter · Lukas Fritsch · Roland Eisendle · Anke Bockreis · Wolfgang Rauch · Michael Kraxner
}

Online publiziert: 10. Juli 2017

(c) Der/die Autor(en) 2017. Dieser Artikel ist eine Open-Access-Publikation.

\begin{abstract}
Zusammenfassung Mit dem Einsatz von Co-Substraten in Faultürmen an Abwasserreinigungsanlagen gelangen unerwünschte Störstoffe in den Prozess, welche aufgrund von Ablagerungen und verstärktem Verschleiß zu erhöhten Betriebskosten führen. Im vorliegenden Beitrag wird die Konzeption und Durchführung von Laborversuchen zur maßgeschneiderten Auslegung eines Partikelabscheiders - im konkreten Fall eines Hydrozyklons - beschrieben. Die Störstoffproblematik bei der CoVergärung von organischen Reststoffen in Abwasserreinigungsanlagen wird trotz eingeschränkter Basisdaten und saisonaler Schwankungen nachvollziehbar für einen Modellfall an einer Versuchsanlage abgebildet.
\end{abstract}

Schlüsselwörter Co-Vergärung · Störstoffe · Partikelabscheidung · Modellbildung
T. Senfter $(\bowtie) \cdot$ L. Fritsch · M. Kraxner Department Umwelt-, Verfahrensund Energietechnik, Management Center Innsbruck (MCI),

Maximilianstraße 2, 6020 Innsbruck, Österreich

thomas.senfter@student.uibk.ac.at

T. Senfter $\cdot$ R. Eisendle

Abwasserverband Zirl und Umgebung,

Meilbrunnen 5, 6170 Zirl, Österreich

Univ.-Prof. Dr.-Ing. A. Bockreis .

Univ.-Prof. DI Dr. W. Rauch

Arbeitsbereich Umwelttechnik,

Universität Innsbruck,

Technikerstraße 13, 6020 Innsbruck,

Österreich
Identification of the influencing variables for the separator design for the selective removal of impurities in the co-fermentation process of organic residues in waste water treatment plants

Abstract Substrates which are used in the co-fermentation process at wastewater treatment plants often contain undesired impurities. These impurities lead to excessive operating costs due to deposits and increased wear and tear. The present paper describes the design and implementation of laboratory experiments for the development of a custom-made particle separator by the geometry of a hydrocyclone for the removal of impurities. The aim is to model the impurities and their physical properties in a laboratory scale. Despite limited basic data and seasonal fluctuations of the feed a comprehensive show case has been derived.

Keywords Co-fermentation .

Impurities - Particle separation .

Modelling

Abkürzungen

$\begin{array}{ll}c & \text { Konzentration }(\mathrm{g} / \mathrm{l}) \\ c_{G} & \text { Konzentration Fraktion Glas }\end{array}$ (g/l)

$c_{\text {ges }} \quad$ Konzentration gesamt (g/l)

$c_{M} \quad$ Konzentration Fraktion Metall (g/l)

$c_{S} \quad$ Konzentration Fraktion Sand $(\mathrm{g} / \mathrm{l})$

$c_{W} \quad$ Strömungswiderstands-

koeffizient

d Durchmesser (mm)

$d_{50} \quad$ Mediandurchmesser $(\mathrm{mm})$

$d_{a} \quad$ Zyklondurchmesser ( $\mathrm{mm}$ )

$d_{e} \quad$ Einlaufdurchmesser des $\mathrm{Zy}$ klons (mm)

$d_{i} \quad$ Tauchrohrdurchmesser des Zyklons (mm) $d_{m} \quad$ Durchmesser der Referenzlinie des Zyklons (mm)

$d_{p} \quad$ Partikeldurchmesser (mm)

$d_{R, a} \quad$ Innendurchmesser des Probenbehälters $(\mathrm{mm})$

$d_{R, i} \quad$ Außendurchmesser des Rühraggregats $(\mathrm{mm})$

$d_{R, m} \quad$ Durchmesser der Referenzlinie des Rührers (mm)

$d^{\prime} \quad$ Partikeldurchmesser bei $Q\left(d_{p}\right)=$ 0,632 (mm)

$F_{A} \quad$ Auftriebskraft (N)

$F_{G} \quad$ Gewichtskraft (N)

$F_{W} \quad$ Widerstandskraft $(\mathrm{N})$

$g$ Erdbeschleunigung $\left(\mathrm{m} / \mathrm{s}^{2}\right)$

$m_{F} \quad$ Masse Feingut (Oberlauffilter) (kg)

$m_{G} \quad$ Masse Grobgut (Unterlauffilter) $(\mathrm{kg})$

$n$ Rührerdrehzahl (1/min)

$n \quad$ Steigung der Verteilungsgeraden $(1 / \mathrm{mm})$

Q Verteilungssumme (\%)

$Q_{G} \quad$ Verteilungssumme Fraktion Glas (\%)

Qges Verteilungssumme gesamt (\%)

$Q_{M} \quad$ Verteilungssumme Fraktion

$\begin{array}{lll} & \text { Metall (\%) } & \\ \text { QS } & \text { Verteilungssumme } & \text { Fraktion }\end{array}$

Sand (\%)

Re Reynoldszahl

$u \quad$ Umfangsgeschwindigkeit $(\mathrm{m} / \mathrm{s})$

$v \quad$ Sinkgeschwindigkeit $(\mathrm{m} / \mathrm{s})$

$z \quad$ Zentrifugalbeschleunigung $\left(\mathrm{m} / \mathrm{s}^{2}\right)$

$\eta \quad$ Abscheidegrad

$\rho_{p} \quad$ Dichte der Partikel $\left(\mathrm{kg} / \mathrm{m}^{3}\right)$

$\rho_{f} \quad$ Dichte der Flüssigkeit $\left(\mathrm{kg} / \mathrm{m}^{3}\right)$

\section{Hintergrund}

1.1 Co-Fermentation

Zur Steigerung der Energieeffizienz werden in Abwasserreinigungsanlagen (ARA) auf der einen Seite verschiedenste Maßnahmen zur Senkung des 
Eigenverbrauchs (z. B. Austausch veralteter Aggregate, Implementierung von Prozessleitsystemen) umgesetzt, andererseits wird auch die Energieproduktion optimiert. Hierzu hat sich in den vergangenen Jahren die Co-Vergärung von organischen Reststoffen als wesentlicher Faktor für die Verbesserung der Energieausbeute etabliert. Nowak und Ebner (2016) geben - ohne Anspruch auf Vollständigkeit - die folgenden typischen Co-Substrate an:

- Küchenabfälle und Speisereste aus der Gastronomie,

- überlagerte Lebensmittel,

- Inhalte von Fettabscheidern,

- Reststoffe von Schlachtbetrieben, aus der Milchverarbeitung oder der Lebensmittelindustrie,

- getrennt gesammelte Bioabfälle aus Haushalten (z. B. „Biotonne“),

- Glycerin aus der Biodieselproduktion.

Der größte Vorteil der Co-Vergärung liegt darin, dass diese durch wenig zusätzlichen Aufwand in bestehenden Faultürmen implementiert werden kann, wodurch freie Faulraumkapazitäten genutzt werden (Bolzonella et al. 2006; Borowski 2015; Cavinato et al. 2013; Edelmann et al. 2000; Nowak und Ebner 2016). Dabei wird die Biogasgewinnung durch die Zugabe von CoSubstrat gesteigert, wobei die gewonnene Energie zur Deckung des Eigenbedarfs sowie zur Einspeisung ins Netz verwendet wird (Bischofsberger et al. 2005).

\subsection{Störstoffe}

Je nach Herkunft des Co-Substrates enthält dieses neben den gewollten organischen Anteilen auch Störstoffe. Diese bestehen, insbesondere bei den getrennt gesammelten Bioabfällen und Speiseresten, aus Knochen, Kernen, Glas, Metall oder Sand. Die Ursache ist zumeist in Fehlwürfen zu finden (Kranert et al. 2002; Kübler et al. 2004; Jank et al. 2016). Durch die Störstoffe entstehen Ablagerungen im Faulturm und erhöhter Verschleiß in Rohrleitungen bzw. Anlagenteilen. Dies wirkt sich negativ auf die Betriebskosten der ARA aus und erfordert eine Abtrennung der Störstoffe, bevor diese in den Faulturm gelangen (Mata-Alvarez et al. 2014; Jank et al. 2015; Rosenwinkel et al. 2015). Auf die Störstoffeigenschaften wirken sich neben der Art der Reststoffe hauptsächlich die vorgeschalteten Aufberei- tungsschritte wie beispielsweise Hammermühlen oder Pressen aus (Hansen et al. 2007; Bernstad et al. 2013). Eine systematische Charakterisierung der Störstoffe wird dadurch erschwert, dass die jeweiligen Stoffströme tageszeitlichen und saisonalen Schwankungen unterliegen (Jank et al. 2016).

\subsection{Störstoffabtrennung mittels Hydrozyklon}

Für die Entfernung der Störstoffe im großtechnischen Maßstab sind die gängigsten Apparate der mechanischen Fest-Flüssig-Trennung, wie beispielsweise Filter oder Zentrifugen, wenig geeignet. Eine vielversprechende Lösung hierfür ist der Einsatz eines Hydrozyklons. Der Vorteil dieses Apparates liegt primär in der einfachen Bauweise, wodurch große Robustheit und lange Standzeiten gegeben sind (Luckert 2004). Wissenschaftliche Untersuchungen zum Abscheideverhalten in Hydrozyklonen reichen zurück bis ca. Mitte des vorigen Jahrhunderts. Einige prägende Personen in diesem Bereich sind Kelsall (1952), Trawinski (1958), Bradley und Pulling (1959), Rietema (1961) oder Svarovsky und Thew (1992). Für die Vorhersage der Trennleistung von Hydrozyklonen wurden verschiedene Modelle und empirisch ermittelte Gleichungen entwickelt. Werden die anhand dieser Gleichungen berechneten Werte mit jenen aus Experimenten verglichen, so kann zusammenfassend festgehalten werden, dass jedes der Näherungsmodelle nur für einen ganz bestimmten Einsatzbereich Gültigkeit aufweist. Folglich sind empirische Versuche begleitend zur theoretischen Auslegung von Hydrozyklonen zu empfehlen (Chen et al. 2000; Krai-

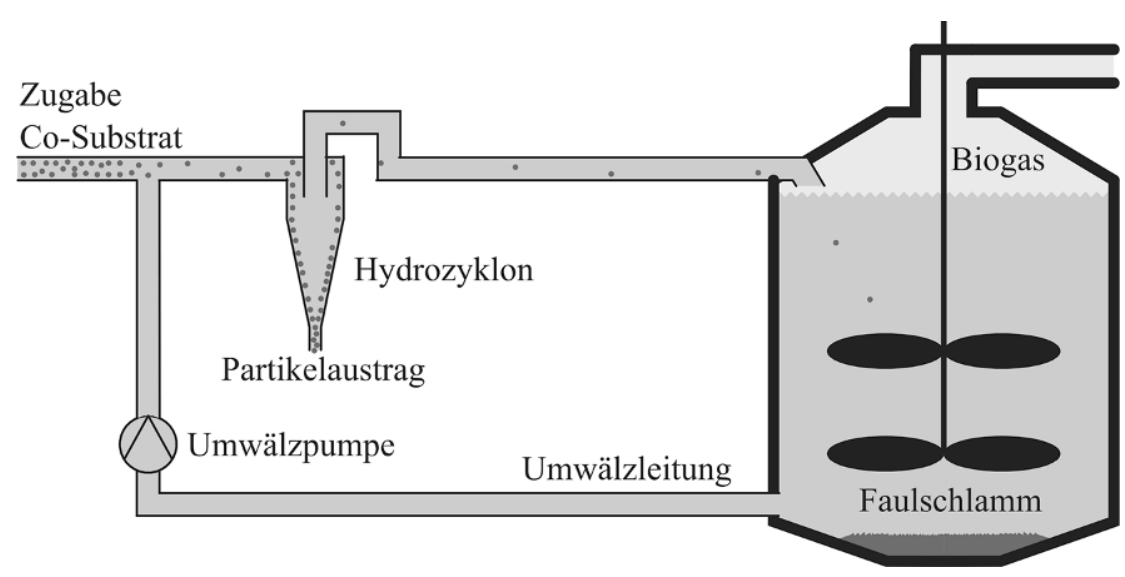

Abb. 1 Schematische Darstellung des Anlagenkonzeptes in der ARA Zirl pech et al. 2006). Für den gegebenen Anwendungsfall im Bereich Co-Vergärung ist dies umso mehr gültig, da das rheologische Verhalten der Fermentationsschlämme sowie der organischen Reststoffe deutliche Unterschiede gegenüber den im Hydrozyklon-Bereich primär untersuchten Wasser-Sand-Systemen aufweist (Baudez et al. 2011).

\section{Zielsetzung und Methodik}

Im vorliegenden Beitrag werden die einzelnen Schritte zur Ermittlung der Einflussgrößen für die Auslegung eines Trennapparates für einen ausgewählten Modellfall beschrieben.

\subsection{Anlagenkonzept und Modellfall}

Zur Auslegung eines geeigneten Trennapparates (in diesem Fall eines Hydrozyklons) sind zuerst die vorliegenden Stoffströme $\mathrm{zu}$ erfassen und die $\mathrm{zu}$ geführte Suspension $\mathrm{zu}$ beschreiben, bevor mit der eigentlichen Auslegung begonnen werden kann. Folgende Faktoren erschweren eine systematische Herangehensweise an die Fragestellung:

- Es liegen nur eingeschränkte Informationen hinsichtlich der Störstoffeigenschaften vor.

- Die Stoffströme in der ARA sowie jener des Co-Substrates unterliegen tageszeitlichen und saisonalen Schwankungen.

Die ARA Zirl (Abwasserverband Zirl und Umgebung), welche für eine Kapazität von 61.500 Einwohnergleichwerten ausgelegt ist, wird als Modellfall ausgewählt. Im Jahr 2013 wurde an der ARA Zirl ein Hydrozyklon in die Umwälzleitung des Faulturms installiert, welcher 


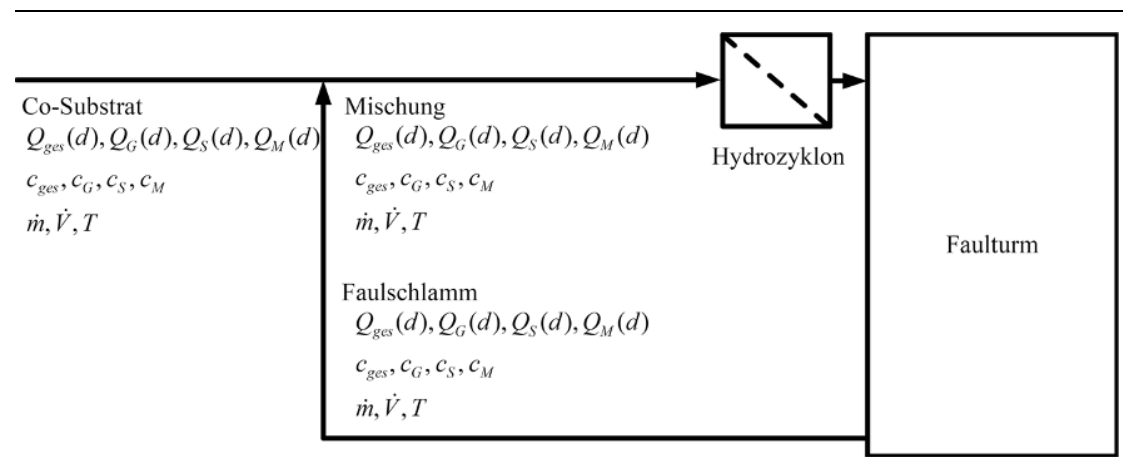

Abb. 2 Vereinfachtes Übersichtsbild mit den vorliegenden Stoffströmen

Störstoffe aus einer Mischung von Faulschlamm und getrennt gesammelten häuslichen und gewerblichen Bioabfällen abtrennt (Eisendle et al. 2014). Eine direkte Abtrennung der Störstoffe aus dem Co-Substrat wäre aufgrund der großen Viskosität nicht möglich. Aus diesem Grund erfolgt vor dem Hydrozyklon die Verdünnung mittels Faulschlamm aus der Umwälzleitung, siehe Abb. 1. Mit diesem Anlagenkonzept und der gewählten Zyklongeometrie können 70-80\% aller Störstoffpartikel größer als $2 \mathrm{~mm}$ abgeschieden werden (Jank et al. 2017).

Angesichts der üblichen Trennleistung von Hydrozyklonen bis in Trennkorngrößenbereiche von wenigen Mikrometern (Schubert 2002) liegt der Schluss nahe, dass das Optimum des eingesetzten Hydrozyklons hinsichtlich Geometrie und Betriebsweise noch nicht erreicht ist. Seitens der Anlagenbetreiber wird als Erfahrungswert angenommen, dass in den Perioden der Zugabe von Co-Substrat ein Mischungsverhältnis von $10 \%$ Co-Substrat mit $90 \%$ Faulschlamm in der Umwälzleitung vorliegt. Für diese Mischung liegt die Viskosität in einem Wertebereich, in welchem eine Partikelabscheidung im Hydrozyklon möglich erscheint.

Bezüglich des Durchflusses im Hydrozyklon wirkt sich die installierte Umwälzpumpe limitierend aus, welche bei der nachträglichen Installation des Hydrozyklons nicht durch eine stärkere Pumpe ersetzt und eigentlich für den Betrieb ohne den Hydrozyklon ausgelegt wurde (Eisendle et al. 2014). Abb. 2

zeigt ein vereinfachtes Fließbild mit jenen Informationen (Korngrößenverteilungen, Konzentrationen), welche zur zielführenden Auslegung eines Abscheiders über die jeweiligen Stoffströme vorliegen sollten.

Der Volumenstrom des Faulschlammes wird mittels eines magnetischinduktiven Durchflusssensors ermittelt, ebenso der Volumenstrom des CoSubstrats. Zu den Korngrößenverteilungen und Konzentrationen der Störstoffe liegen einige wenige Werte vor, welche gemäß Jank et al. (2016) ermittelt wurden, siehe Tab. 1.

Ebenso liegen seitens der Anlagenbetreiber Richtwerte für die Gesamtkorngrößenverteilung der Störstoffe im Faulschlamm sowie im Co-Substrat vor, jedoch ohne Zuordnung zu den jeweiligen Materialien Glas/Sand bzw. Metall (siehe Abb. 3).

Der Vergleich von Tab. 1 und Abb. 3 mit Abb. 2 zeigt, dass nur ein Bruchteil der für die umfassende Beschreibung des vorliegenden Systems notwendigen Informationen vorhanden ist. Die daraus folgenden Annahmen und Vereinfachungen werden in den Abschn. 2.2 und 2.3 erläutert.

\subsection{Störstoffe}

Die Bilanzierung von Gewichtskraft, Widerstandskraft und Auftriebskraft in einem stationären Sedimentationsprozess von Partikeln im Schwerefeld ist in Gl. (1) gezeigt:

$$
F_{G}=F_{W}+F_{A}(\text { in N })
$$

Tab. 1 Richtwerte Störstoffeigenschaften ARA Zirl

\begin{tabular}{|l|l|l|}
\hline Störstoff & Mediandurchmesser $d_{50}$ in $\mathrm{mm}$ & Konzentration c in $\mathrm{g} / \mathrm{l}$ \\
\hline Glas/Sand im Faulschlamm & 0,2 & 0,3 \\
\hline Metall im Faulschlamm & 0,6 & 0,1 \\
\hline Glas/Sand im Co-Substrat & 0,8 & 10 \\
\hline Metall im Co-Substrat & 1,2 & 5 \\
\hline
\end{tabular}

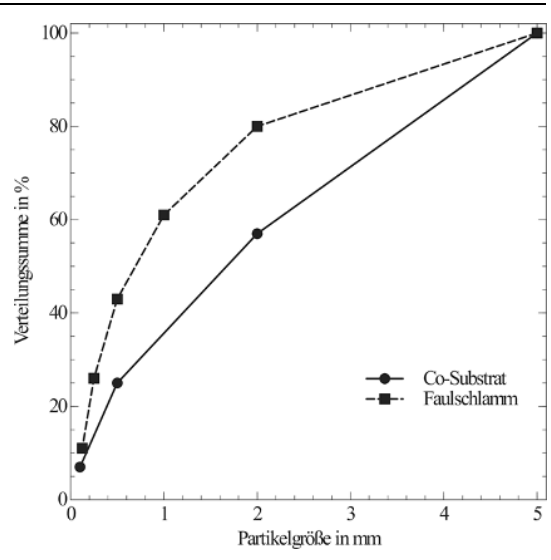

Abb. 3 Richtwerte Korngrößenverteilung der Störstoffe in Faulschlamm und Co-Substrat

Daraus abgeleitet ergibt sich nach Gl. (2) die allgemeine Sedimentationsgeschwindigkeit (Stieß 2008):

$$
v=\sqrt{\frac{4}{3} \cdot \frac{d_{p} \cdot z}{c_{W}(R e)} \cdot\left(\frac{\rho_{p}}{\rho_{f}}-1\right)}\left(\text { in } \frac{\mathrm{m}}{\mathrm{s}}\right)
$$

Die Störstofffraktionen Glas und Sand werden $\mathrm{zu}$ einem gemeinsamen Material Glas/Sand (GS) zusammengefasst. Diese Vereinfachung wird damit gerechtfertigt, dass die wichtigsten Parameter zur Bestimmung der Sedimentationsgeschwindigkeit im Zentrifugalfeld gemäß Gl. (2) der Partikeldurchmesser sowie die Dichtedifferenz zwischen Partikel und Fluid sind. Der Aufbereitungsprozess für das Co-Substrat umfasst $u$. a. eine Zerkleinerung mittels einer Hammermühle. $\mathrm{Ob}$ es sich beim zu zerkleinernden Gut um Glas oder Stein handelt, wirkt sich hinsichtlich des Zerkleinerungsergebnisses kaum aus (Gil und Arauzo 2014).

Als weitere Vereinfachung wird angenommen, dass es sich beim Material der Störstoffe um Aluminium handelt. Die Dichte von Aluminium beträgt $2710 \mathrm{~kg} / \mathrm{m}^{3}$, während jene von Stahl im Bereich von $7700 \mathrm{~kg} / \mathrm{m}^{3}$ liegt. Somit ist ein Störstoffpartikel aus Aluminium für die Auslegung des Trennapparates der ungünstigere Fall gegenüber eines Stahlpartikels, welches gemäß Gl. (2) leichter abgeschieden wird. Zur Berücksichtigung des für den Abscheideprozess „ungünstigen“ Falles wird die Partikelgröße aus Abb. 3 mit dem Faktor 0,75 skaliert.

Existieren von einer Schüttung keine Informationen für die gesamte Korngrößenverteilung, so können mittels Standardverteilungen aus den (weni- 
gen) bekannten Informationen Verteilungssummen genähert werden (Stieß 2008). Die gängigsten Standardverteilungen sind:

- Potenzverteilung (nach Gates, Gaudin und Schuhmann; DIN 66143)

- Logarithmische Normalverteilung (DIN 66144)

- RRSB-Verteilung (nach Rosin, Rammler, Sperling und Bennet; DIN 66145)

Für alle drei Verteilungsfunktionen wird eine Eignungsanalyse durchgeführt.

\subsection{Viskosität}

Für die Auslegung des Partikelabscheiders ist die Viskosität des Mediums eine wesentliche Einflussgröße, vgl. Gl. (2). Je größer die Viskosität ist, desto geringer ist die radiale Sedimentationsgeschwindigkeit der Partikel im Abscheideraum und umso schlechter ist somit auch der Abscheidegrad (Kawatra et al. 1996). Da Faulschlämme kein Newtonsches Fließverhalten aufweisen, kann auch kein Zahlenwert für die Viskosität angegeben werden (Slatter 1997, Seyssiecq et al. 2003). Ebenso ist die Reproduzierbarkeit von rheologischen

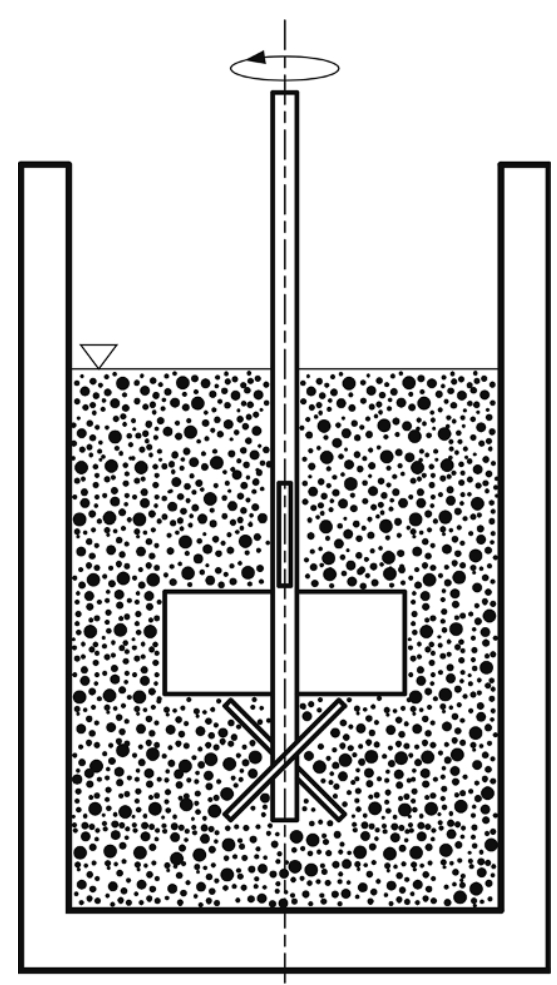

Abb. 4 Rührer-Messsystem für den Vergleich des rheologischen Verhaltens von Schlämmen
Untersuchungen oftmals wenig zufriedenstellend. Moshage (2004) berichtet beispielsweise von Abweichungen um den Faktor 28 bei Schlämmen mit gleichem Feststoffgehalt. Darüber hinaus ist sich die Literatur hinsichtlich des zu verwendenden Messsystems uneinig. Moshage (2004) empfiehlt z. B. ein konzentrisches Zylindermesssystem zur Analyse der Viskosität. Dies bedingt jedoch eine Siebung des Schlammes vor Beginn der Messung, wodurch eine Veränderung der Zusammensetzung und folglich der Viskosität gegeben ist. Insgesamt sind rheologische $\mathrm{Da}$ ten für Faulschlämme aus der Literatur nur schwer vergleichbar, da kein standardisiertes Messprotokoll und keine einheitliche Handhabung der Proben vorgegeben ist (Eshtiaghi et al. 2013). Eine Alternative zum Zylindermesssystem, bei welchem keine Vorbehandlung der Probe notwendig ist, stellt ein Rührer-Viskosimeter dar (Reviol 2010). Wie in Abb. 4 ersichtlich, sind Feststoffpartikel bis $\mathrm{zu}$ einer bestimmten Größe aufgrund des Spaltes zwischen Probenbehälter und Rührer keine Ursache für mechanische Beschädigungen des Messsystems. Da jedoch bei diesem Messsystem die Scherrate nicht aus den Geometrieparametern berechnet werden kann, ist eine Angabe der Viskosität in mPas nicht möglich. Aus diesem Grund wird beim Rührer-Messsystem üblicherweise das eingetragene Moment bestimmt und je nach Anwendungsfall mit Referenzmessungen verglichen (Mezger 2010).

Die Probennahme und Probenteilung stellt neben der Wahl eines geeigneten Messsystems eine weitere Herausforderung bei den rheologischen Untersuchungen dar. Insbesondere die tageszeitlichen und saisonalen Schwankungen in den Stoffströmen an den Abwasserreinigungsanlagen bedingen eine angepasste Anzahl an Wiederholungen für die Messungen. Für die Untersuchung des Modellfalls ARA Zirl werden über einen Zeitraum von 2 Monaten 10 Proben des Faulschlammes und des Co-Substrates genommen. Untersucht wird jeweils eine Mischung aus $10 \%$ Co-Substrat und $90 \%$ Faulschlamm. Um Einflüsse aufgrund der Probenteilung $\mathrm{zu}$ vermeiden, wird jede dieser Proben wiederum $10 \mathrm{Mal}$ vermessen. Schließlich liegt eine Referenzprobe vor, welche mittels des Verlaufes des eingebrachten Drehmoments beschrieben werden kann. Zur Herstellung einer Modellsubstanz mit ähnli- chem rheologischen Verhalten werden verschiedene Rheologieadditive hinsichtlich ihrer Eignung untersucht.

\subsection{Versuchsanlage}

Die selektive Abscheidung der Störstoffe erfordert eine maßgeschneiderte Auslegung des Trennapparates. Hierfür sind zusätzlich zu den theoretischen Überlegungen empirische Trennversuche notwendig. Die Durchführung dieser Versuche direkt an der ARA ist schwierig, da die Zugänglichkeit zu den Stoffströmen mitunter eingeschränkt ist und eine große Ausfallsicherheit im ARA-Betrieb gefordert ist. Aus diesem Grund wird eine Versuchsanlage zur reproduzierbaren Durchführung von Experimenten entwickelt. Eine wesentliche Anforderung an die Versuchsanlage ist, dass alle Betriebsparameter definiert und überwacht werden können. Zur Bilanzierung des Hydrozyklons müssen die Partikelmassen im Zulauf sowie jene im Unterlauf (abgetrennt) und Oberlauf (nicht abgetrennt) bekannt sein. In der Literatur üblicherweise eingesetzte Versuchsanlagenkonzepte (Braun 1989; Neesse et al. 2015) beruhen auf der Bestimmung der Konzentration im Ober- und Unterlauf anhand von Online-Dichtemessungen oder Teilprobennahmen. Beide Varianten weisen Einschränkungen hinsichtlich der Flexibilität (Kalibrierung) oder der Reproduzierbarkeit (Messbereich, Empfindlichkeit gegenüber Feststoffpulsation) auf. Die im vorliegenden Beitrag gezeigte gravimetrische Bestimmung des Abscheidegrads mittels Oberlauf- und Unterlauffilter führt zu Ergebnissen mit geringer Schwankungsbreite.

\section{Ergebnisse}

\subsection{Störstoffe}

Für die Eignungsanalyse bezüglich der Standardverteilungen werden die bekannten Korngrößen aus Abb. 3 in jedes Netz der in Abschn. 2.2 angeführten Verteilungsfunktionen eingetragen. Jene Standardverteilung wird als geeignet für die Beschreibung der Korngrößenverteilung angesehen, bei welcher die eingetragenen Punkte bestmöglich auf einer Geraden liegen. Für den Modellfall ARA Zirl erweist sich die RRSB-Verteilung als geeignet. Mittels der Funktion gemäß Gl. (3) kann somit 
Hier steht eine Anzeige.

Springer 
Hier steht eine Anzeige.

Springer 

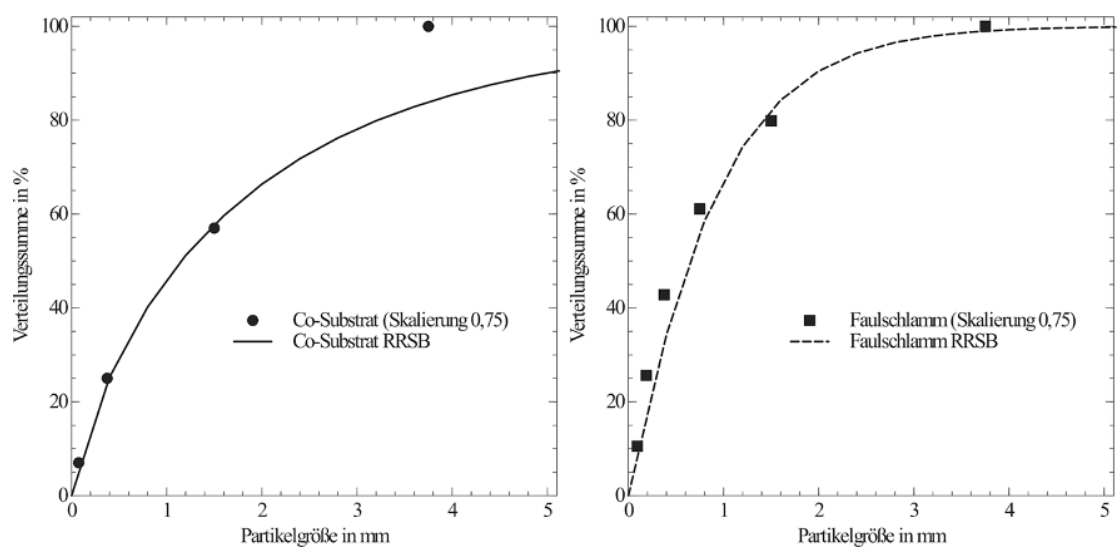

Abb. 5 RRSB-Verteilung für die skalierten Gesamt-Störstoffe im Co-Substrat (links) und im Faulschlamm (rechts)

die Korngrößenverteilung bestimmt werden (Stieß 2008):

$$
Q\left(d_{p}\right)=1-e^{-\left(\frac{d_{p}}{d^{\prime}}\right)^{n}}
$$

Abb. 5 zeigt die mit dem Faktor 0,75 skalierten Korngrößen aus Abb. 3 im Vergleich mit den Verteilungsfunktionen. Für die RRSB-Verteilung der Störstoffe im Co-Substrat gelten folgende Parameter:

- $d^{\prime}=1,8 \mathrm{~mm}$

- $n=0,82$

Die Störstoffe im Faulschlamm können gemäß Gl. (3) und folgenden Parametern beschrieben werden:

- $d^{\prime}=0,9 \mathrm{~mm}$

- $n=1,07$

Die Störstoffkonzentrationen in Abhängigkeit von den jeweiligen Materialien sind in Tab. 1 angegeben. Zusätzlich erfolgt eine Zuordnung zu den Stoffströmen Co-Substrat und Faulschlamm.

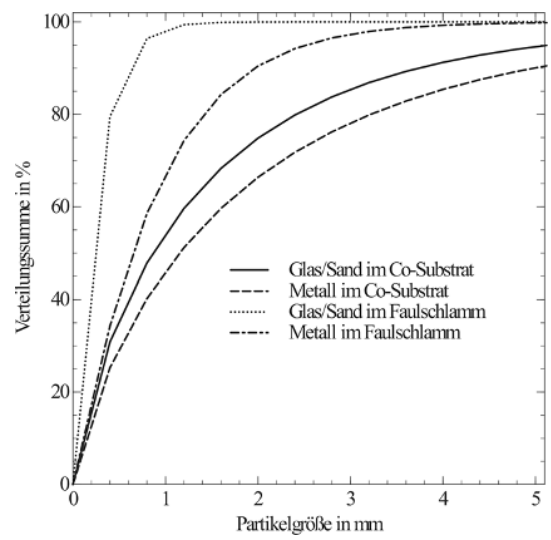

Abb. 6 Korngrößenverteilungen mit Zuordnung zu Material und Stoffstrom
Unter der Annahme, dass die Verteilungsbreite innerhalb eines Stoffstromes sowohl für Glas/Sand als auch Metall gleich ist, kann bei bekanntem $d_{50}$ aus Tab. 1 für jede Störstofffraktion und jeden Stoffstrom eine entsprechende Korngrößenverteilung angegeben werden. Das Ergebnis ist in Abb. 6 dargestellt.

Ist es möglich, einen funktionierenden Zentrifugalabscheider für die Störstoffe Glas/Sand im Faulschlamm zu entwickeln, so wird dieser auch die größeren Partikel aus den anderen Materialien bzw. Stoffströmen abscheiden. Für Laborversuche zur Optimierung des Partikelabscheiders müssen folglich Labor-Störstoffe ausgewählt werden, welche die Eigenschaften der Fraktion Glas/Sand im Faulschlamm aufweisen. Sind neben der Partikelgröße noch Faktoren wie Verschleißminimierung und minimales Aufquellen des Materials zu berücksichtigen, so eignen sich Marmorpartikel besonders zur Abbildung der Störstoffe im Labor. Diese sind in den Partikelgrößenbereichen gemäß Tab. 2 erhältlich.

Durch Mischung der Partikel im entsprechenden Massenverhältnis kann die Korngrößenverteilung aus Abb. 6 reproduzierbar für Laborversuche hergestellt werden.

Tab. 2 Partikelgrößenbereiche und Artikelbezeichnungen für die Versuchspartikel

Bezeichnung Korngrößenbereich in $\mu \mathrm{m}$
Carolith 0-0,2 0-200 Carolith $0,2-0,5 \quad 200-500$ Carolith 0,5-1 500-1000

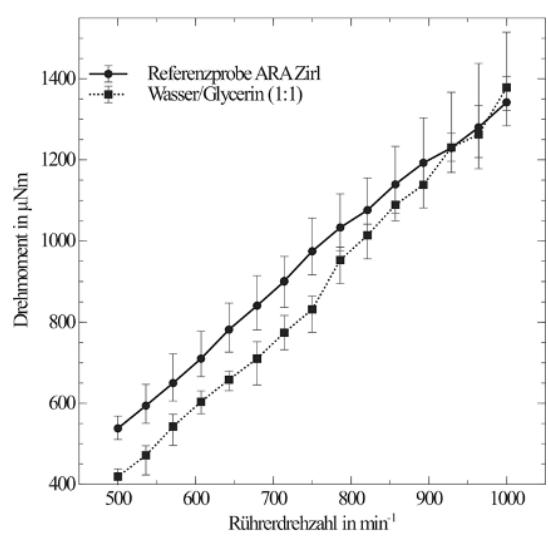

Abb. 7 Rheologisches Verhalten der Referenzprobe (ARA Zirl) und Nachbildung mittels Wasser/Glycerin (die Fehlerbalken geben jeweils den gemessenen Minimalund Maximalwert an)

\subsection{Viskosität}

Das Ergebnis der Eignungsanalyse für die Rheologieadditive ist in Tab. 3 ersichtlich.

Für alle angeführten Rheologieadditive wurden Messungen in verschiedenen Mischungsverhältnissen mit Wasser durchgeführt. Abb. 7 zeigt den Verlauf des Drehmoments der Referenzprobe sowie jenes der Modellflüssigkeit Wasser/Glycerin im Massenverhältnis von 1:1. Die Messung erfolgte mittels des Rotationsviskosimeters RheolabQC der Firma Anton Paar.

Für Gemische aus Wasser und Glycerin sind, in Abhängigkeit vom Massenverhältnis, Viskositätswerte bekannt. Für das Gemisch aus 50 \% Glycerin und $50 \%$ Wasser wird von Haynes (2015) eine Viskosität von $6 \mathrm{mPas}$ angegeben, wobei eine Scherratenabhängigkeit nicht berücksichtigt wird. Bei diesem Wert bleibt jedoch die Empfehlung von Mezger (2010) unberücksichtigt, beim Rührermesssystem nur das eingetragene Moment anzugeben. Eine allgemein gültige rheologische Charakterisierung von Fermentationsschlämmen ist folglich nicht möglich, weshalb je nach verwendetem Messsystem zu untersuchen ist, ob dieses für die jeweilige Fragestellung geeignet ist (Eshtiaghi et al. 2013). Zur Evaluierung, ob die im RührerMesssystem auftretenden Scherraten in einem ähnlichen Bereich liegen wie jene im Hydrozyklon, wird eine Referenzlinie gemäß Abb. 8 betrachtet. Diese liegt bei der angestrebten Bauform des Hydrozyklons im Zentrum des Einlaufes und wird mit dem mittleren 
Tab. 3 Eignungsanalyse Rheologieadditive

\begin{tabular}{l|l|l|l|}
\hline $\begin{array}{l}\text { Substanz } \\
\text { Hochdisperse } \\
\text { Kieselsäure }\end{array}$ & Produktbezeichnung & Vorteil & Nachteil \\
\hline $\begin{array}{l}\text { Glycerin/1,2,3- } \\
\text { Propandiol }\end{array}$ & - & $\begin{array}{l}\text { Reproduzierbarkeit, } \\
\text { Industrieprodukt }\end{array}$ & $\begin{array}{l}\text { Trübung, Einrühren in } \\
\text { Wasser notwendig }\end{array}$ \\
\hline $\begin{array}{l}\text { Zucker/ } \\
\text { Polysaccharid }\end{array}$ & Xanthan 200 mesh & $\begin{array}{l}\text { Preiswertes Industrie- } \\
\text { produkt }\end{array}$ & - \\
\hline
\end{tabular}
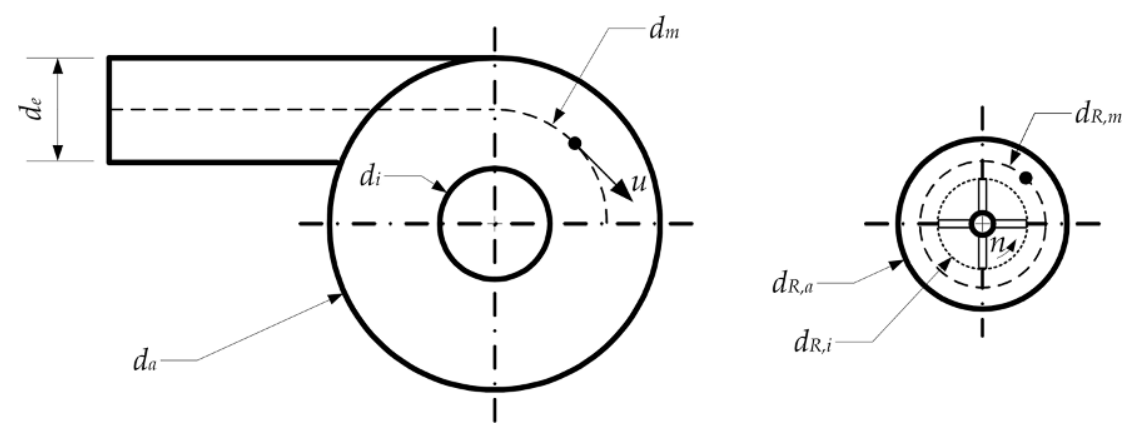

Abb. 8 Referenzlinie Hydrozyklon (links) und Rührer (rechts) zum Vergleich der auftretenden Zentrifugalbeschleunigung

Durchmesser $d_{m}$ bezeichnet. Die Referenzlinie des Rührer-Messsystems liegt auf halber Spaltweite zwischen den Rührerblättern und der Innenwand des Probenbehälters (bezeichnet als $d_{R, m}$ ).

Für die Zentrifugalbeschleunigung gilt:

$$
\left.z=\frac{2 \cdot u^{2}}{d} \text { (in } \frac{\mathrm{m}}{s^{2}}\right)
$$

mit:

$$
u=n \cdot d \cdot \pi\left(\text { in } \frac{\mathrm{m}}{\mathrm{s}}\right)
$$

Die Geometrieparameter sind in Tab. 4 ersichtlich. Sie basieren für den Hydrozyklon auf einer Grobauslegung gemäß Rietema (1961) und für den Rührer auf den Systemen ST24 und CC39 der Firma Anton Paar.

Die Unter- und Obergrenze der

Tab. 4 Geometrieparameter des Labor-Hydrozyklons und Rührers

\begin{tabular}{|l|l|}
\hline Parameter & Wert in mm \\
\hline$d_{e}$ & 28 \\
\hline$d_{a}$ & 100 \\
\hline$d_{i}$ & 28 \\
\hline$d_{m}$ & 72 \\
\hline$d_{R, a}$ & 39 \\
\hline$d_{R, i}$ & 24 \\
\hline$d_{R, m}$ & 31,5 \\
\hline
\end{tabular}

Tab. 5 Vergleich von Zentrifugalbeschleunigungenund Geschwindigkeiten

\begin{tabular}{|l|l|l|}
\hline System & $\begin{array}{l}\text { Parameter } n, \\
u\end{array}$ & $\begin{array}{l}\text { Beschleunigung } \\
z\left(\mathrm{~m} / \mathrm{s}^{2}\right)\end{array}$ \\
\hline Rührer & $\begin{array}{l}n= \\
500 \mathrm{~min}^{-1}\end{array}$ & 43,17 \\
\hline Rührer & $\begin{array}{l}n= \\
1000 \mathrm{~min}^{-1}\end{array}$ & 172,72 \\
\hline Hydrozyklon & $\begin{array}{l}u= \\
1,24 \mathrm{~m} / \mathrm{s}\end{array}$ & 43,17 \\
\hline Hydrozyklon & $\begin{array}{l}u= \\
2,49 \mathrm{~m} / \mathrm{s}\end{array}$ & 172,72 \\
\hline
\end{tabular}
die entsprechende Geschwindigkeit im Zyklon berechnet werden (siehe Tab. 5).
Der berechnete Wertebereich der Umfangsgeschwindigkeit $u$ auf der Referenzlinie des Hydrozyklons entspricht typischen Werten bei der praktischen Anwendung des Abscheiders. Auf Basis der getätigten Annahmen und Berechnungen erscheint das ermittelte rheologische Verhalten geeignet, um die im Zyklon vorherrschenden Sedimentationsvorgänge zu beschreiben.

\subsection{Versuchsanlage}

Abb. 9 zeigt den Aufbau der Versuchsanlage. Die Zirkulationspumpe MP1 führt die Modellflüssigkeit dem Hydrozyklon zu. Vor dem Hydrozyklon erfolgt die Aufgabe der Feststoffe durch die Partikeldosierung NC1. Für die Regelung des Einlaufvolumenstroms wird das Signal des Durchflusssensors FICl verwendet. Die Drucksensoren PI1 und PI2 bestimmen den Druckverlust und somit den Energiebedarf für den Betrieb des Hydrozyklons. Der Unterlaufvolumenstrom ist frei einstellbar und wird mittels des Durchflusssensors FIC2 und der Unterlaufpumpe MP2 geregelt. Die Temperaturmessung TI1 dient dazu, Temperaturänderungen und somit eine Änderung der Viskosität auszuschließen.

Die Bestimmung der abgetrennten und nicht abgetrennten Partikelmasse erfolgt gravimetrisch durch Trocknung und Wiegen des Oberlauf- sowie Unterlauffilters nach jedem Versuch. Die Versuchsanlage zur Abbildung des Modellfalls ARA Zirl wird anhand der in Tab. 6 beschriebenen Parameter ausgelegt.

Im Folgenden wird beispielhaft ein Ergebnis gezeigt, welches an der Versuchsanlage generiert wurde. Untersucht wird der Einfluss des Unterlaufvolumenstromes auf den Abscheidegrad des Labor-Hydrozyklons mit den in Abb. 10 ersichtlichen Geometrieparametern.

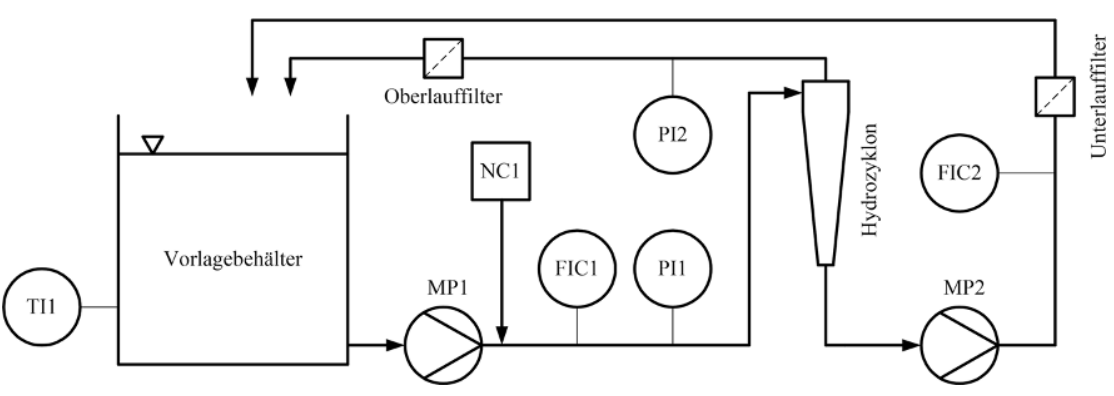

Abb. 9 Schematischer Aufbau der Hydrozyklon-Versuchsanlage 


\begin{tabular}{l|l|l|l}
\hline \multicolumn{2}{l}{ Tab. 6} & Auslegungsgrundlagen für die Versuchsanlage & \\
\hline Parameter & Wert min & Wert typ & Wert max \\
\hline Einlaufvolumenstrom & $1 \mathrm{~m}^{3} / \mathrm{h}$ & $5 \mathrm{~m}^{3} / \mathrm{h}$ & $40 \mathrm{~m}^{3} / \mathrm{h}$ \\
\hline Unterlaufvolumenstrom & $0,05 \mathrm{~m}^{3} / \mathrm{h}$ & $0,2 \mathrm{~m}^{3} / \mathrm{h}$ & $5 \mathrm{~m}^{3} / \mathrm{h}$ \\
\hline Druck & $0 \mathrm{bar}$ & $1,5 \mathrm{bar}$ & $4 \mathrm{bar}$ \\
\hline Partikelmassenstrom & $20 \mathrm{~g} / \mathrm{h}$ & $1500 \mathrm{~g} / \mathrm{h}$ & $10.000 \mathrm{~g} / \mathrm{h}$
\end{tabular}

Tab. 7 Versuchsparameter Unterlaufuntersuchung

\begin{tabular}{l|l|}
\hline $\begin{array}{l}\text { Parameter } \\
\text { Versuchspartikel }\end{array}$ & Wert \\
\hline Einlaufvolumenstrom & $5 \mathrm{~m}^{3} / \mathrm{h}$ \\
\hline Unterlaufvolumenstrom 1 & $0,1 \mathrm{~m}^{3} / \mathrm{h}(2 \%)$ \\
\hline $\begin{array}{l}\text { Unterlaufvolumenstrom 2 } \\
\text { Unterlaufvolumenstrom 3 }\end{array}$ & $0,2 \mathrm{~m}^{3} / \mathrm{h}(4 \%)$ \\
\hline $\begin{array}{l}\text { Wiederholungen pro Unter- } \\
\text { laufvolumenstrom }\end{array}$ & $0,4 \mathrm{~m}^{3} / \mathrm{h}(8 \%)$ \\
\hline $\begin{array}{l}\text { Anzahl Versuche gesamt } \\
\text { Kontinuierliches Medium }\end{array}$ & 27 \\
\hline $\begin{array}{l}\text { Partikelkonzentration im } \\
\text { Einlauf }\end{array}$ & Wasser \\
\hline
\end{tabular}

Weitere Versuchsparameter sind in Tab. 7 gelistet.

Die Bestimmung des Abscheidegrads erfolgt gemäß Gl. (6) aus der Partikelmasse im Ober- und Unterlauffilter:

$$
\eta=\frac{m_{G}}{m_{G}+m_{F}}
$$

Die Abhängigkeit des Abscheidegrads vom Unterlaufvolumenstrom ist in Abb. 11 dargestellt. Ein erhöhter Unterlaufvolumenstrom hat eine bessere Abscheidung zur Folge. Dies resultiert daraus, dass bei mehr Unterlauf eine bessere Geschwindigkeitsfortpflanzung im Abscheideraum stattfindet und da-
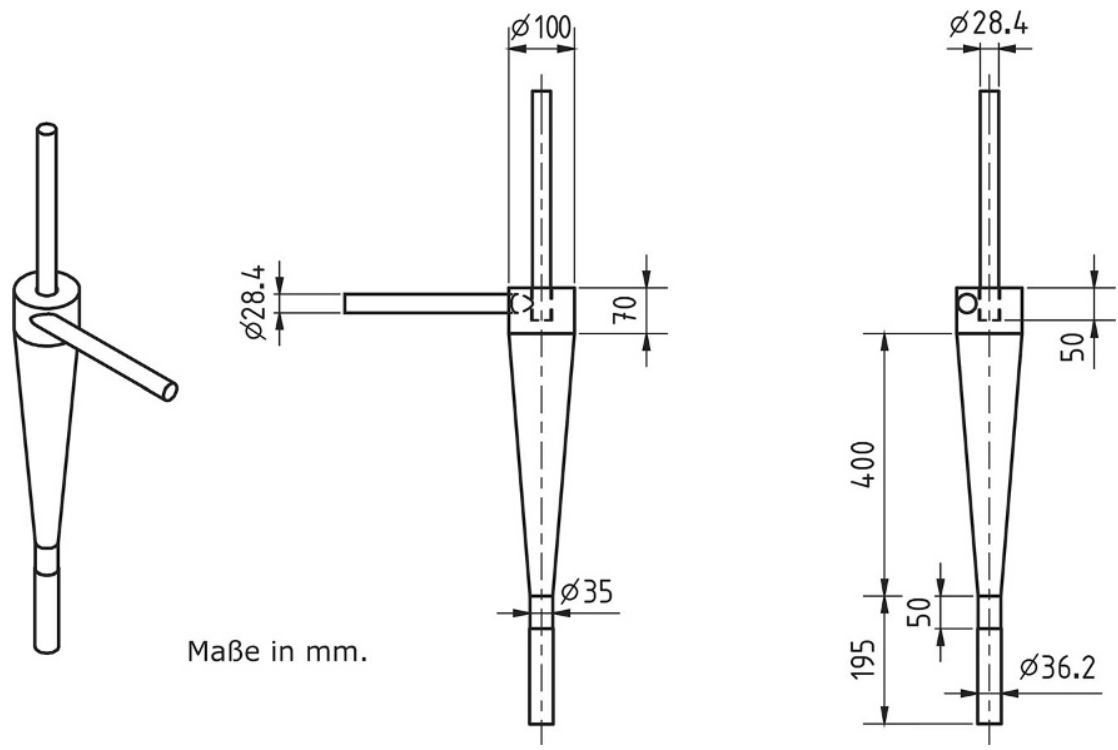

\section{Zusammenfassung und Ausblick}

Neben den vielen Vorteilen (z. B. gesteigerte Energieausbeute), die bei der Co-Vergärung von organischen Reststoffen an Abwasserreinigungsanlagen entstehen, stellt die ungewollte Einbringung von Störstoffen in den Faulturm ein wesentliches Problem für die Anlagenbetreiber dar. Eine mögliche Abhilfe für dieses Problem soll die Installation eines Hydrozyklons in die Umwälzleitung des Faulturmes bringen. Im vorliegenden Beitrag wurde gezeigt, wie aus einem beschränkten Datensatz zur Beschreibung der Ausgangssituation im Co-Vergärungsprozess auf nachvollziehbare Art und Weise eine Abbildung des Problems für Laborversuche erfolgen kann. Die Laborversuche sollen schließlich die Basis für eine optimierte Auslegung eines Partikelabscheiders darstellen. Informationslücken hinsichtlich der Partikelgrößenverteilungen wurden mittels Standardverteilungen und der Annahme eines „ungünstigen“ Falles überbrückt. Bei der Bestimmung des rheologischen Verhaltens des Schlammes erweist sich ein Rührer-Messsystem als geeignete Methodik, um die Viskosität mit Hilfe von Rheologieadditiven nachzubilden. An einer eigens entwickelten Versuchsanlage werden die Optimierungen am Hydrozyklon durchgeführt. Die ersten Ergebnisse am Laborzyklon zeigen, dass dem Unterlaufvolumenstrom eine wesentliche Bedeutung für die Höhe des erzielbaren Abscheidegrads zufällt. Für die praktische Anwendung bedeutet dies, dass hinsichtlich der weiteren Behandlung des anfallenden (partikelreichen) Unterlaufes vertiefende Überlegungen (z. B. nachgeschalteter Abscheider, Rückführung des Unterlaufes in den Prozess) notwendig sind. Zusammenfassend ist festzuhalten, dass die Stoffströme in der ARA hinsichtlich ihrer Zusammensetzung tageszeitlichen und saisonalen Schwankungen unterliegen. Dies ist eine weitere Herausforderung beim Versuch, die komplexen Zustände in der ARA-Praxis im Labor abzubilden. Dieser Umstand wurde berücksichtigt, indem über einen Zeitraum von zwei Monaten an 10 verschiedenen Tagen Proben genommen wurden und jede dieser Proben 10-mal vermessen wurde. Somit liegen hinter jedem der in Abb. 7 dargestellten Punkte 100 Messungen.

Abb. 10 Geometrieparameter Labor-Hydrozyklon 
Die Optimierungen des Labor-Hydrozyklons werden anhand von Feldversuchen an der ARA Zirl überprüft. Hierzu wird ein optimierter Hydrozyklon in die Umwälzleitung integriert und nach erfolgreicher Inbetriebnahme bezüglich Abscheidegrad und Energiebedarf evaluiert.

Open access funding provided by University of Innsbruck and Medical University of Innsbruck.

Danksagung Die Bearbeitung der Fragestellung im Themenbereich „Hydro- zyklone zur Störstoffabtrennung in der Co-Fermentation" erfolgt in einem Kooperationsprojekt mit folgenden Partnern: MCI (Forschungsbereich Fluids \& Mechanics), BioTreaT GmbH, Universität Innsbruck (Arbeitsbereich Umwelttechnik), hydro-IT GmbH, PORR Bau GmbH, HTL Anichstraße (Abteilung Maschinenbau), HTL Bau und Design (Abteilung Tiefbau) und sechs Tiroler Abwasserverbänden (Zirl und Umgebung, Innsbrucker Kommunalbetriebe, Achental-Inntal-Zillertal, Pitztal, Telfs und Umgebung sowie WörglKirchbichl). Allen Partnern sei an dieser Stelle herzlich gedankt.
Open Access Dieser Artikel wird unter der Creative Commons Namensnennung 4.0 International Lizenz (http:// creativecommons.org/licenses/by/4. $0 /$ deed.de) veröffentlicht, welche die Nutzung, Vervielfältigung, Bearbeitung, Verbreitung und Wiedergabe in jeglichem Medium und Format erlaubt, sofern Sie den/die ursprünglichen Autor(en) und die Quelle ordnungsgemäß nennen, einen Link zur Creative Commons Lizenz beifügen und angeben, ob Änderungen vorgenommen wurden.
Baudez, J.C., Markis, F., Eshtiaghi, N., Slatter, P (2011): The rheological behaviour of anaerobic digested sludge, Water Research 45:5675-5680. DOI: 10.1016/j.watres.2011.08.035

Bernstad, A., Malmquist, L., Truedsson, C. Jansen, J.C. (2013): Need for improvement in physical pretreatment of source-separated household food waste. Waste Management 33:746-754, DOI: 10.1016/j.wasman.2012.06.012 Bischofsberger, W., Dichtl, N., Rosenwinkel, K.H., Seyfried, C.F., Böhnke, B. (2005): Anaerobtechnik, Springer, Berlin Heidelberg

Bolzonella, D., Battistoni, P., Susini, C., Cecchi,

F. (2006): Anaerobic codigestion of waste ac tivated sludge and OFMSW: the experiences of Viareggio and Treviso plants (Italy). Water Science and Technology 53:203-211. DOI: 10.2166/wst.2006.251

Borowski, S. (2015): Co-digestion of the hydromechanically separated organic fraction of municipal solid waste with sewage sludge. Journa of Environmental Management 147:87-94. DOI 10.1016/j.jenvman.2014.09.013

Bradley, D., Pulling, D.J. (1959): Flow pattern in a hydraulic cyclone and their interpretation in terms of performance, Transactions of the Institution of Chemical Engineers 37:34-45

Braun, T. (1989): Theoretische und experimentelle Untersuchungen des Einflusses de Feststoffkonzentration und der Partikelgrößenverteilung auf das Trennverhalten von Hydrozyklonen, Dissertation, TU Carolo-Wilhelmina Braunschweig

Cavinato, C., Bolzonella, D., Pavan, P., Fatone, F., Cecchi, F. (2013): Mesophilic and thermophilic anaerobic co-degestion of waste activated sludge and source sorted biowaste in pilot- and full-scale reactors, Renewable Energy 55:260-265. DOI: 10.1016/j.renene.2012.12.044 Chen, W., Zydek, N., Parma, F. (2000): Evaluation of Hydrocyclone Models for Practical Applications, Chemical Engineering Journal 80:295-303. DOI: 10.1016/S1383-5866(00)00105-2

Edelmann, W., Engeli, H., Gradenecker, M. (2000): Co-digestion of organic solid waste and sludge from sewage treatment. Water Science and Technology 41:213-221. DOI:

Eisendle, R., Niederkirchner, A., Ebner, C. (2014): Energieproduktion an der Kläranlage: Neuartige Zyklontechnik könnte Lösung für die Abtrennung von Störstoffen bringen. Abwasserverband Zirl und Umgebung. http://www.avzirl. at/xoffi_files/file_24_1029.pdf. Gesehen 1. Mai 2017

Eshtiaghi, N., Markis, F., Yap, S.D., Baudez, J.C.

Slatter, P. (2013): Rheological characterization of municipal sludge: A review. Water Research 47:5493-5510. DOI: 10.1016/j.watres.2013.07.001 Gil, M., Arauzo, I. (2014): Hammer mill operating and biomass physical conditions effects on particle size distribution of solid pulverized biofuels. Fuel Process Technology 127:80-87. DOI 10.1016/j.fuproc.2014.06.016

Hansen, T.I., Jansen, J.C., Davidsson, A., Christensen, T.H. (2007): Effects of pre-treatment technologies on quantity and quality of sourcesorted municipal organic waste for biogas recovery. Waste Management 27:398-405. DOI 10.1016/j.wasman.2006.02.014

Haynes, W.M. (2015): Handbook of chemistry and physics, CRC Press, Boca Raton

Jank, A., Müller, W., Schneider, I., Gerke, F., Bockreis, A. (2015): Waste Separation Press (WSP): A mechanical pretreatment option for organic waste from source separation. Waste Management 39:71-77. doi:10.1016/j.wasman. 2015.02.024

Jank, A., Müller, W., Waldhuber, S., Gerke, F. Ebner, C., Bockreis, A. (2016): Impurities in pretreated biowaste for co-digestion: A determination approach. Waste Management 52:96-103. DOI: 10.1016/j.wasman.2016.03.055

Jank, A., Müller, W., Waldhuber, S., Gerke, F., Ebner, C., Bockreis, A. (2017): Hydrocyclones for the separation of impurities in pretreated biowaste. Waste Management 64:12-19. DOI: 10.1016/j.wasman.2017.03.001

Kawatra, S.K., Bakshi, A.K., Rusesky, M.T. (1996): Effect of viscosity on the cut (d50): size of hydrocyclone classifiers. Minerals Engineering 9:881-891. DOI: 10.1016/0892-6875(96)00079-9 Kelsall, D.F. (1952), A study of the motion of solid particles in a hydraulic cyclone, Transactions of the Institution of Chemical Engineers 30:87-108.

Kraipech, W., Chen, W., Dyakowski, T., Nowakowski, A. (2006): The performance of the empirical models on industrial hydrocyclone design International Journal of Mineral Processing 80 : 100-115. DOI: 10.1016/j.minpro.2005.02.005

Kranert, M., Graul, S., Hillebrecht, K. (2002) Untersuchungen zur Bestimmung von Mineralgehalten in Bioabfällen und Gärrückständen. Müll und Abfall, 612-617

Kübler, H., Hentschke, C., Bakenecker, U. (2004): Bestimmung von Schwerstoffen in $\mathrm{Ab}$ fall-Pulpen. Müll und Abfall, 581-584

Luckert, K. (2004): Handbuch der mechanischen Fest-Flüssig-Trennung. Vulkan, Essen Mata-Alvarez, J., Dosta, J., Romero-Güiza, M.S., Fonoll, X., Peces, M., Astals, S. (2014): A critical review on anaerobic co-digestion achieve- ments between 2010 and 2013. Renewable and Sustainable Energy Reviews 36:412-427. DOI: 10.1016/S0960-8524(00)00023-7

Mezger, T. (2010): Das Rheologie Handbuch, Vincentz Network, Hannover

Moshage, U. (2004): Rheologie kommunale Klärschlämme - Messmethoden und Praxisrelevanz. Schriftenreihe des Instituts für Siedlungswasserwirtschaft der TU Braunschweig, Heft Nr. 72, https://www.tu-braunschweig.de/isww/ schriftenreihe/heft72. Gesehen 1. Mai 2017

Neesse, T., Dueck, J., Schwemmer, H., Farghaly, M. (2015): Using a high pressure hydrocyclone for solids classification in the submicron range. Minerals Engineering 71:85-88. DOI: 10.1016/j.mineng.2014.10.017

Nowak, O., Ebner, C. (2016): Verwertung organischer Reststoffe in Faulbehältern kommunaler Kläranlagen. Österr Wasser- und Abfallw 68:108-117. DOI: 10.1007/s00506-016-0294-74 Reviol, T. (2010): Experimentelle und numerische Untersuchungen eines modifizierten Propeller-Viskosimeters zur Bestimmung de Fließeigenschaften nicht-Newtonscher Medien mit inhomogenem Charakter, Dissertation, Technische Universität Kaiserslautern

Rietema, K. (1961): Performance and design of hydrocyclones, Chemical Engineering Science 15.298-325. DOI: 10.1016/0009-2509(61)85033-1 Rosenwinkel, K.H., Kroiss, H., Dichtl, N., Seyfried, C.-F., Weiland, P. (2015): Anaerobtechnik. Abwasser-, Schlamm- und Reststoffbehandlung, Biogasgewinnung. Springer Vieweg, Berlin, Heidelberg

Schubert, H. (2002): Handbuch der Mechanischen Verfahrenstechnik. WILEY-VCH, Weinheim

Seyssiecq, I. (2003): State-of-the-art: rheological characterisation of wastewater treatment sludge. Biochemical Engineering Journal 16:41-56. DOI: 10.1016/S1369-703X(03)00021-4

Slatter, PT. (1997): The rheological characterisation of sludges. Water Science and Technology 36:9-18. DOI: 10.1016/S0273-1223(97)00663-X Stieß, M. (2008): Mechanische Verfahrenstechnik - Partikeltechnologie 1, Springer, Berlin Svarovsky, L., Thew, M.T. (1992): Hydrocyclones. Analysis and Applications. Kluwer Academic Publishers, Dortrecht

Trawinski, H. (1958): Näherungssätze zur Berechnung wichtiger Betriebsdaten für Hydrozyklone und Zentrifugen. Chemie Ingenieur Technik 30:85-95. DOI: 10.1002/cite.330300206 